\title{
Atomistic Insights on the Wear/Friction Behavior of Nanocrystalline Ferrite During Nanoscratching as Revealed by Molecular Dynamics
}

\author{
A. T. AlMotasem ${ }^{1,2}$ (1) J. Bergström ${ }^{1} \cdot$ A. Gåård ${ }^{1} \cdot$ P. Krakhmalev $^{1}$ \\ L. J. Holleboom ${ }^{1}$
}

Received: 23 January 2017/ Accepted: 29 May 2017/Published online: 29 June 2017

(c) The Author(s) 2017. This article is an open access publication

\begin{abstract}
Using embedded atom method potential, extensive large-scale molecular dynamics (MD) simulations of nanoindentation/nanoscratching of nanocrystalline (nc) iron have been carried out to explore grain size dependence of wear response. MD results show no clear dependence of the frictional and normal forces on the grain size, and the single-crystal (sc) iron has higher frictional and normal force compared to nc-samples. For all samples, the dislocation-mediated mechanism is the primary cause of plastic deformation in both nanoindentation/nanoscratch. However, secondary cooperative mechanisms are varied significantly according to grain size. Pileup formation was observed in the front of and sideways of the tool, and they exhibit strong dependence on grain orientation rather than grain size. Tip size has significant impact on nanoscratch characteristics; both frictional and normal forces monotonically increase as tip radii increase, while the friction coefficient value drops by about $38 \%$. Additionally, the increase in scratch depth leads to an increase in frictional and normal forces as well as friction coefficient. To elucidate the relevance of indentation/scratch results with mechanical properties, uniaxial tensile test was performed for nc-samples, and the result indicates the existence of
\end{abstract}

Electronic supplementary material The online version of this article (doi:10.1007/s11249-017-0876-y) contains supplementary material, which is available to authorized users.

A. T. AlMotasem

ahmed.al-asqalani@kau.se; a.almotasem@science.au.edu.eg

1 Department of Mechanical and Materials Engineering, Karlstad University, Universitetsgatan 2, 65637 Karlstad, Sweden

2 Department of Physics, Faculty of Science, Assiut University, Assiut 71516, Egypt both the regular and inverse Hall-Petch relations at critical grain size of $110.9 \AA$. The present results suggest that indentation/scratch hardness has no apparent correlation with the mechanical properties of the substrate, whereas the plastic deformation has.

Keywords Atomistic · Polycrystalline iron · Scratch hardness $\cdot$ Wear $\cdot$ Dislocations $\cdot$ Twinning

\section{Introduction}

Understanding wear, friction and mechanical properties of a material at nanoscale is crucial for further development in technological applications. Experimentally, nanoindentation and nanoscratching techniques are commonly used for nanoscale mechanical testing as they can provide accurate information of hardness, friction and wear. As a compliment to experimental technique, atomistic modeling becomes a powerful tool to deepen the understanding of wear and failure modes of materials at the atomic scale. In the literature, numerous studies of nanoscratching of metals are available both theoretically and experimentally and a comprehensive review can be found in $[1,2]$.

Molecular dynamics simulation has been used to investigate nanoscale machining and the factors governing the nanomachining process: tip geometry, machining speed, rake angle and surface roughness. However, most of these simulations usually adopt defect-free monocrystalline structures as the work material [3-9]. On the other hand, most engineering materials exist in polycrystalline forms and mechanical properties such as flow stress, yield stress and hardness of metals and alloys $[10,11]$ dramatically scale with grain size. Thus, grain size in polycrystalline structures is a controlling factor for material properties and material 
responses to deformation. For example, it has been shown that grain refinement to the nanometer scale leads to an increased yield stress Hall-Petch (H-P) relationship, while further refinement led to inverse H-P $[12,13]$. The influence of grain boundaries (GBs) during nanomachining has been extensively studied [14-18]; nevertheless, most of the considered samples are either nc-fcc metals, nc-diamond or nczinc blend ceramics but rarely for nc-bcc materials.

For instance, Shi et al. [15] performed MD simulations to investigate the effect of grain size and nanomachining parameters of polycrystalline copper. It was discovered that for all cutting conditions simulated, the polycrystalline structure requires smaller cutting forces compared with the monocrystalline structure. The authors attributed this behavior to the reduction in material strength with grain refinement. They also verified that the behavior of frictional and normal forces for different grains is coupled with the associated failure mode, i.e., $(\mathrm{H}-\mathrm{P})$ or inverse $(\mathrm{H}-\mathrm{P})$ as verified by tensile testing.

Mishra et al. [18] simulated the wear of a nanocrystalline silicon carbide substrate by tools with a rounded end. They demonstrated that the primary mechanism for nanoscale wear of silicon carbide is GBs sliding and the compatible stress is accommodated by nucleation of partial dislocations, void formation and grains pullout. Furthermore, they compared the results with sc-silicon carbide wear response and demonstrated that nc-silicon carbide is more pliable to wear due to GB sliding.

With respect to bcc iron, several theoretical studies about nanoscratching of iron single crystal exist. For instance, in the earlier work by Mulliah et al. [19], it has been shown that both hardness and deformation mechanism show anisotropic dependence. Later, using the embedded atom method EAM potential and a pyramidal indenter, $\mathrm{Lu}$ and coworkers [20] studied, both by experiment and simulation, the nanoscratch behavior of (100) and (112) bcc$\mathrm{Fe}$ and showed that the atomic movement resistance is higher along close-packed directions.

Recently, Gao et al. [21, 22] have systematically studied the nanoscratching of single crystal by carrying out a series of MD simulations using the Mendelev potential and spherical tip. It has been shown that the wear of iron single crystal is significantly influenced by the orientation of the machined surface and the deformation mechanism of the samples during scratching was dominated by dislocation slip and nc-iron [23]

In the present work, we investigated the grain size dependence of nanoscratching characteristics with particular focus on the deformation mechanisms coupled to the grain size. Additionally, we checked the influence of other scratch parameters such as tip radii and scratch depth. By exploring this study, we can find optimized conditions for improved wear resistance of nc-iron.

\section{Computational Technique}

Polycrystalline iron samples with different grain sizes were constructed following the Voronoi tessellation method [24], implemented within the Atomsk tool [25]. Five samples with average grain sizes, $58.8<D<141.2 \AA$, were built by randomly distributing seeds in a box having a size of $350 \times 210 \times 170 \AA^{3}$. Subsequently, bcc lattice grains with random misorientations were generated from the seeds. The box was periodic in the lateral $-x$ and $-y$ directions, and the two bottommost layers of the workpiece were fixed. The next two layers were thermostat layers to dissipate generated heat during scratching. A rigid, hemispherical and diamond carbon tool with $R=50 \AA$ was used in this work. In order to investigate the influence of the geometrical shape of the tip on the wear and friction results, four different tools with $R=25,35,45,50,55$ and $60 \AA$ in radius have been considered.

In the first stage, overlapped atoms within $0.7 \AA$ distances were deleted, and then, the conjugate gradient method was used to carry out energy minimization to eliminate the initial undesirable artificial defects which may arise from constructing models. In the second stage, the system was thermally equilibrated to $300 \mathrm{~K}$ and $0 \mathrm{GPa}$ pressure using the Nose-Hoover isobaric/isothermal NPT ensemble to reach a stress-free state.

The nanoscratching procedure was carried out as follows: (1) The tip is pushed downward to the workpiece at velocity $30 \mathrm{~m} / \mathrm{s}$ until it reached the prosperities depth; although this velocity is very high compared to indentation experiments, it is sufficient to observe deformation behavior and defect evolution, (2) the system is equilibrated using a microcanonical (NVE) ensemble for 100 ps, followed by (3) tip sliding over the workpiece with velocity $100 \mathrm{~m} / \mathrm{s}$ along $-x$ direction as illustrated in Fig. 1.

The interaction between $\mathrm{Fe}-\mathrm{Fe}$ atoms was described by the potential, denoted as potential 2 in the original paper, developed by Mendelev et al. [26], based on an embedded atomic method (EAM). This potential was developed by fitting to the first principle forces obtained in a model liquid configuration and was verified to successively reproduce mechanical and thermal properties of bcc iron. The interaction between $\mathrm{Fe}$ and $\mathrm{C}$ was described by the purely repulsive Lennard-Jones pair potential with a shifted cutoff radius $4.2 \AA[22,27]$, while the interaction among $\mathrm{C}-\mathrm{C}$ atom was omitted.

In the present work, the identification of dislocations was made via dislocation extraction algorithm (DXA) [28], while the structural defects were analyzed by the Crystal Analysis Tool (CAT) [29, 30]. In this method, the adaptive common neighbor analysis, coordination number and centrosymmetric parameters are used to distinguish bcc 

the MD simulation model of the nanoscratching of nc-iron. Blue atoms are crystalline diamond; yellow atoms are bcc iron, while gray atoms mark grain boundaries and free surfaces. (For interpretation of the references to color in this figure legend, the reader is referred to the web version of this article.) (Color figure online)
Fig. 1 Schematic diagram of

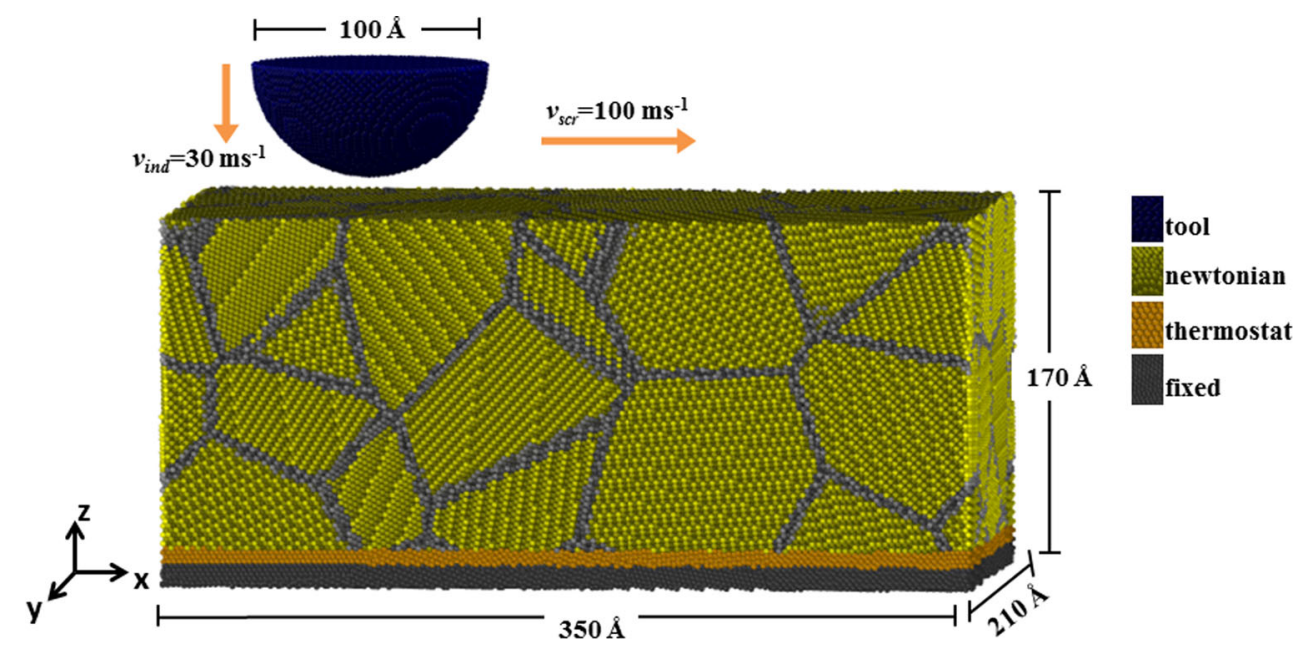

and non-bcc atoms. Then, the neighbors of non-bcc atoms fulfilling the criterion of both coordination number and centrosymmetric parameter are determined and classified according to Table (1) in Ref. [31].

\section{Results and Discussion}

\subsection{Indentation Stage}

Prior to scratching stage, MD nanoindentation simulation was conducted to confirm the direct contact between the tip and workpiece. At first, the tip was placed vertically upward relative to the workpiece surface $(z=0)$ so that the distance between the lowest point of the indenter and the workpiece surface was $10 \AA$. Then, the tip was pushed downward along $z$ direction with indentation velocity $30 \mathrm{~m} / \mathrm{s}$ until the tip reached indentation depth $d=25 \AA$. The indentation force was calculated by summing up the vertical component of force on each atom in the tip. Figure 2 displays the force-displacement curves corresponding to different grain sizes as well as iron single crystal for the sake of comparison. Note that the indentation force starts to build up at distance close to the cutoff radius of the repulsive potential around $4.2 \AA$. It is interesting to see that the peak maximum indentation force varies for both polycrystalline and single-crystal iron even though the maximum indentation depth was the same for all samples. However, in general the single-crystal iron exhibits the largest indentation force. The reason for this behavior might be due to the grain boundary and grain orientation as well as the mechanical properties having highly anisotropic behavior. The analysis of the force-displacement curve for single crystal shows that, at shallow penetration depth $(d<7 \AA)$, the curve is within the elastic regime. According to theory, the indentation force, in the elastic regime, is related to the penetration depth $d$ by

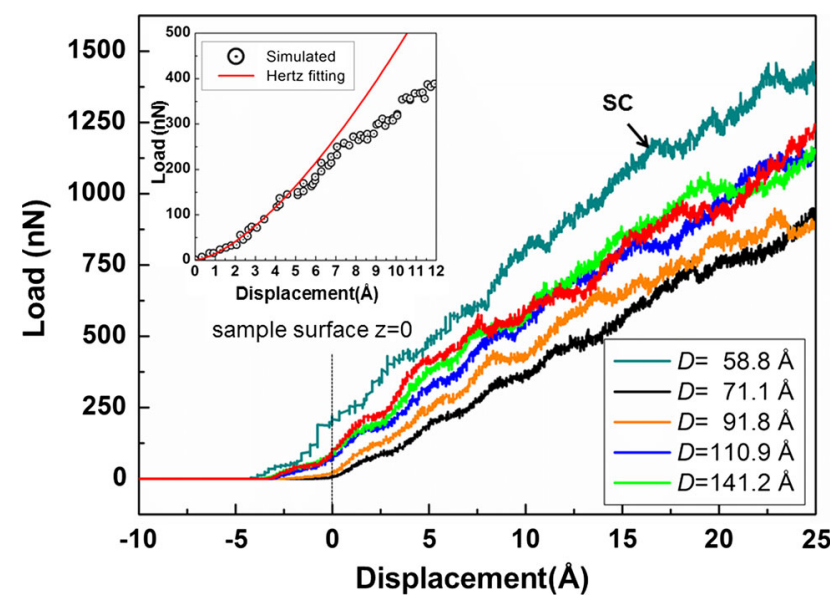

Fig. 2 Simulated load-displacement curves of nanoindentation of polycrystalline $\mathrm{Fe}$ for different grain size. The inset represents the load-displacement curve within elastic regime of single-crystal sample

$F=\frac{4}{3} \sqrt{R} E^{*} d^{3 / 2}$

where $R$ is the radius of the tip and $E^{*}$ the reduced Young's modulus defined as

$\frac{1}{E^{*}}=\frac{1-v_{\mathrm{w}}^{2}}{E_{\mathrm{w}}}+\frac{1-v_{\mathrm{t}}^{2}}{E_{\mathrm{t}}}$

$E$ and $v$ are the Young's modulus and Poisson's ration of the workpiece and tip, respectively. The value of $E_{\mathrm{t}}$ is infinity as the tip is considered rigid, the value of $v_{\mathrm{W}}$ is 0.3 , and the value of $E_{\mathrm{w}}$ is calculated as the average of values of $E$ corresponding to low index planes predicted by the current used interatomic potential [26]. A comparison between the simulation result of single crystal and the force calculated by Eq. (1) exhibits a good agreement as plotted in Fig. 2(inset). 
Prior to the nanoindentation stage, a number of both interior grain and GBs dislocations have been observed by the end of annealing at $300 \mathrm{~K}$ as shown in Fig. 3a, c. We noticed that the presence of GBs dislocations in the asprepared sample is slightly affected by the annealing time. The inhomogeneity of GBs dislocation distribution among different grain boundaries may be attributed to differences of grain boundary structures and orientations. Several MD results have shown that the value of nucleation stress of GBs dislocations varies greatly with the grain boundary structure and inclination angles [32-34]. As the indentation depth was further increased, the plastic regime initiates. We noticed that the insipient plasticity of the nc-iron occurs earlier compared to sc-iron as GBs dislocations play a key role in plasticity incipient of the nanocrystalline material.

The atomic level analysis of deformation mechanisms by dislocation extract algorithm (DXA) reveals that both dislocation nucleation and annihilation are the underlying deformation mechanisms. In the case of sample with larger grain size, the onset of dislocation nucleation occurs directly below the surface of the workpiece. As the indenter further penetrates, the dislocations glide within the grain interior and at the same time remained attached to the tip/workpiece contact; see Fig. 3b. In contrast, in the sample with smaller grains, the nucleated dislocations either immediately annihilate at the GBs to form voids or interact with preexisting GBs dislocations. Thus, grain boundaries act as an obstacle for dislocation extension as displayed in Fig. 3d.
The material response during nanoindentation can be quantified by the indentation hardness, defined as

$H_{\text {ind }}=\frac{F_{\text {ind }}}{A_{\mathrm{p}}^{\text {ind }}}$

where $F_{\text {ind }}$ is the indentation force and $A_{\mathrm{p}}^{\text {ind }}$ is the projected contact area during nanoindentation. The values of $A_{\mathrm{p}}^{\text {ind }}$ were determined during the MD simulation following the procedure proposed in Refs. [22, 35]. In this approach, only atoms lying within the spherical shell of radius $R+r_{\mathrm{c}}$ are counted as contact atoms; see Fig. 4.

$A_{\mathrm{p}}^{\mathrm{ind}}=\pi \sigma^{2} \sum_{i \in R+r_{\mathrm{c}}} \cos \theta_{i}$

$A_{\mathrm{p}}^{\mathrm{scr}}=\pi \sigma^{2} \sum_{i \in R+r_{\mathrm{c}}} \sin \theta_{i}$

Figure 5 depicts the variation of the projected contact area with the displacement. Obviously, $A_{\mathrm{p}}^{\text {ind }}$ increases monotonically, while the tip penetrates the samples. The results of the contact area were used to calculate the indentation hardness according to Eq. (3). Clearly, the value of $H_{\text {ind }}$ increases with increasing grain size, as depicted in Fig. 6b, and all nanocrystalline samples have smaller $H_{\text {ind }}$ value compared to the single-crystal sample. The results are compliant with the theoretical studies [23, 36-38] on nanoindentation of nanocrystalline materials. The decrease in the $H_{\text {ind }}$ with decreasing grain size can be attributed to the increase in grain boundary fraction and interaction between dislocations and GBs.
Fig. 3 MD snapshots of dislocations determined by (DXA) analysis of the dislocation network observed at the maximum indentation depth of samples with grain size 141.2 left columns and $58.8 \AA$, right column. a, c before indentation, $\mathbf{b}, \mathbf{d}$ after the tip reaches the maximum depth. The arrows mark the preexisting dislocations, while circles denote the dislocations resulted from nanoindentation
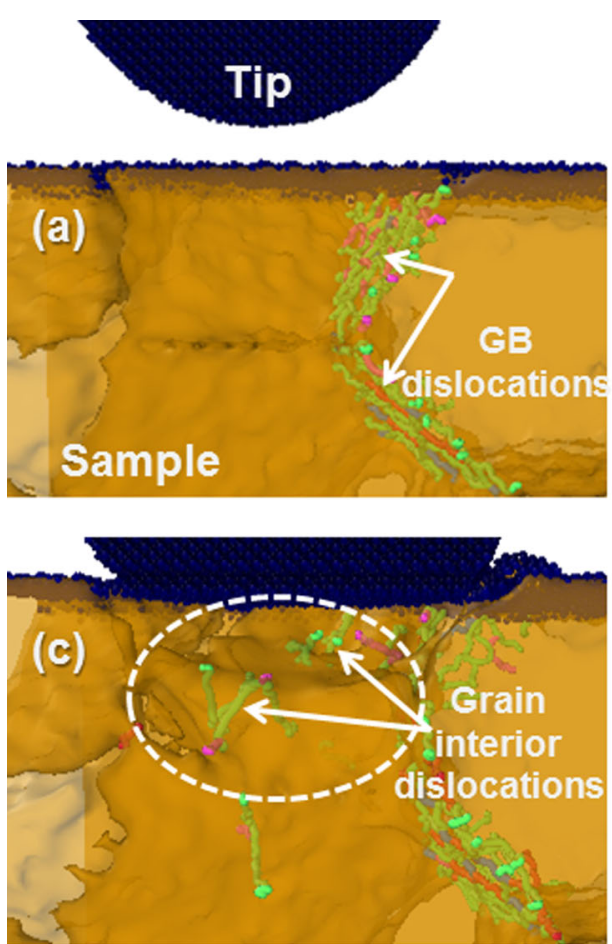
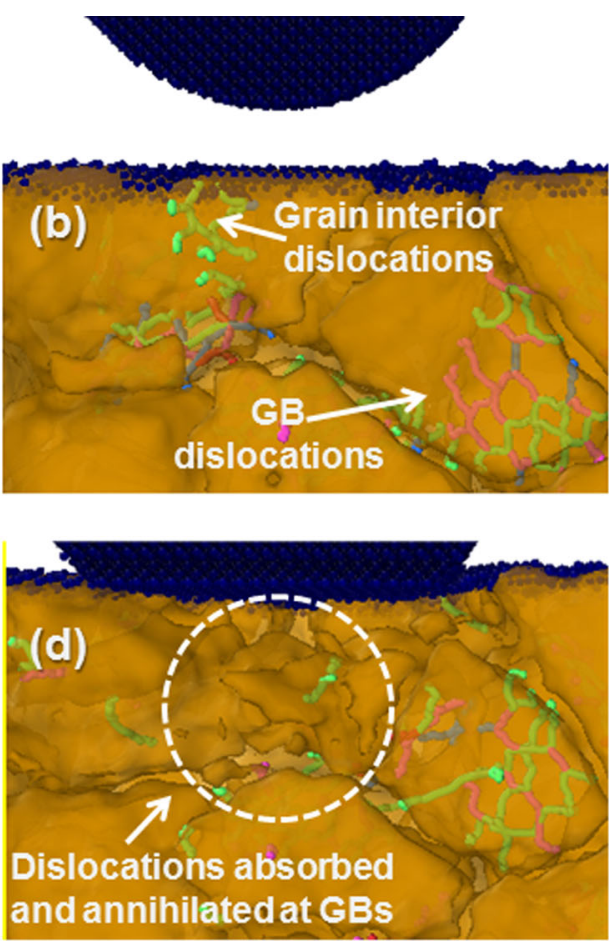


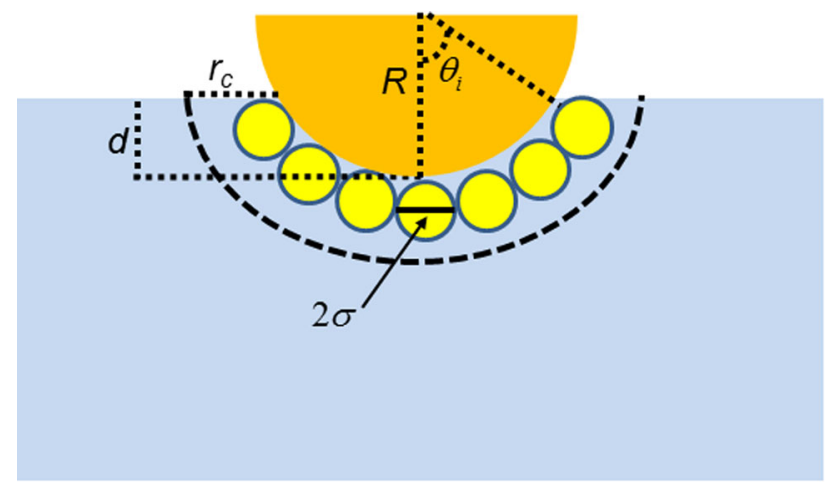

Fig. 4 Schematic diagram showing the calculation of contact area during simulation. $R$ is the tip radius, $r_{\mathrm{c}}$ is the cutoff radius taken to be $4.2 \AA, d$ is the scratch depth, and $\sigma$ is the radius of contact atoms and is given by $1.41 \AA$

\subsection{Scratching Stage}

\subsubsection{Plastic Deformation During the Scratching Stage}

During the scratching, disparate plasticity mechanisms were observed either concurrently and/or sequentially. However, in general the plasticity of all samples was initiated by dislocation nucleation and their movement. Similar to the nanoindentation stage, the two common types of dislocations which are well known for bcc iron were observed, namely $1 / 2\langle 111\rangle$ and $\langle 100\rangle$ as shown in Fig. 7.

Fig. 5 Evolution of the projected contact area during nanoindentation stage; contact atoms from workpiece are colored in yellow (Color figure online)
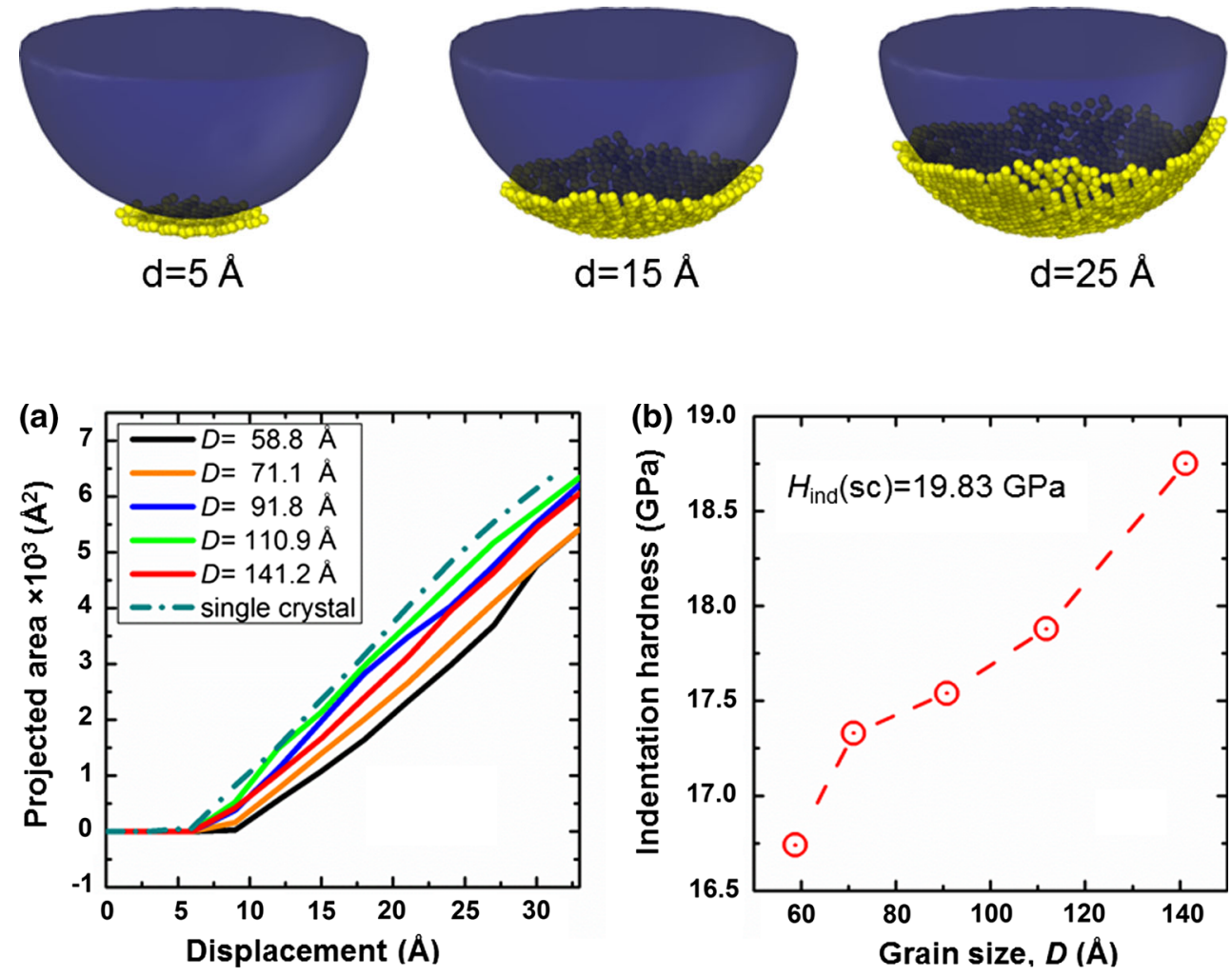

Fig. 6 Evolution of the projected contact area during nanoindentation stage (a), and plot of indentation hardness as a function of grain size (b)
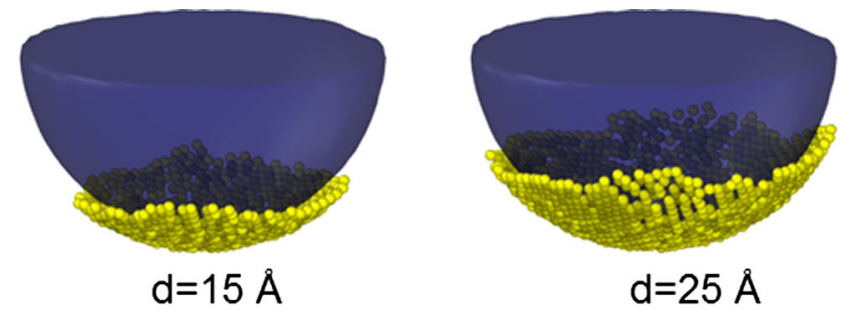

Depending on the grain size, two distinct deformation behaviors were observed. First, in the case of large grains, for instance the sample with grain size $D=141.2 \AA$ as shown in Fig. 7, the dislocations nucleate below the sample surface and then glide within the grain interior. As the tip slides, these dislocations may interact to form a dislocation network or interact with grain boundary dislocations. Further tool advancement leads to several cooperative processes, a dislocation freely migrating to the grain boundary (if not annihilated with another dislocation) and absorbed which causes grain boundary broadening and a transition from planar to non-planar GBs. With continuous sliding, also, dislocation pileup occurred due to confinement of dislocations in a small distance; consequently, a subgrain may be formed. Besides, the dislocations annihilation results in formation of permanent interstitials/voids nanoclusters as shown in Fig. 7a, b. The generation of vacancies and interstitials has been observed during scratching of single-crystal bcc iron (see Ref. [21]). The latter process has been explained by Marian et al. [39]. Using MD simulations, they showed that under high stress, a sudden propagation of a dislocation becomes rough, and therefore, the line becomes rugged, leading to formation of lattice defects such as vacancies and interstitial clusters.

Secondly, for small grain sizes, similar to large grains, the plasticity initiates as dislocation nucleation. However, due to small grain size and small distance between the sliding tip and the position of the grain boundary, the

$d=15 \AA$ 
Fig. 7 Snapshots showing different deformation behaviors during nanoscratching for samples a, b $D=141.2 \AA$, c, d $D=58.8 \AA$ A. Coloring due to common neighbor analysis (CNA), blue represents lattice atoms and gray non-lattice, surface and grain boundary atoms. The bcc atoms of some grains are omitted for exposing grain interior. Numbers shown in (c) denote grains will merge during scratching (Color figure online)
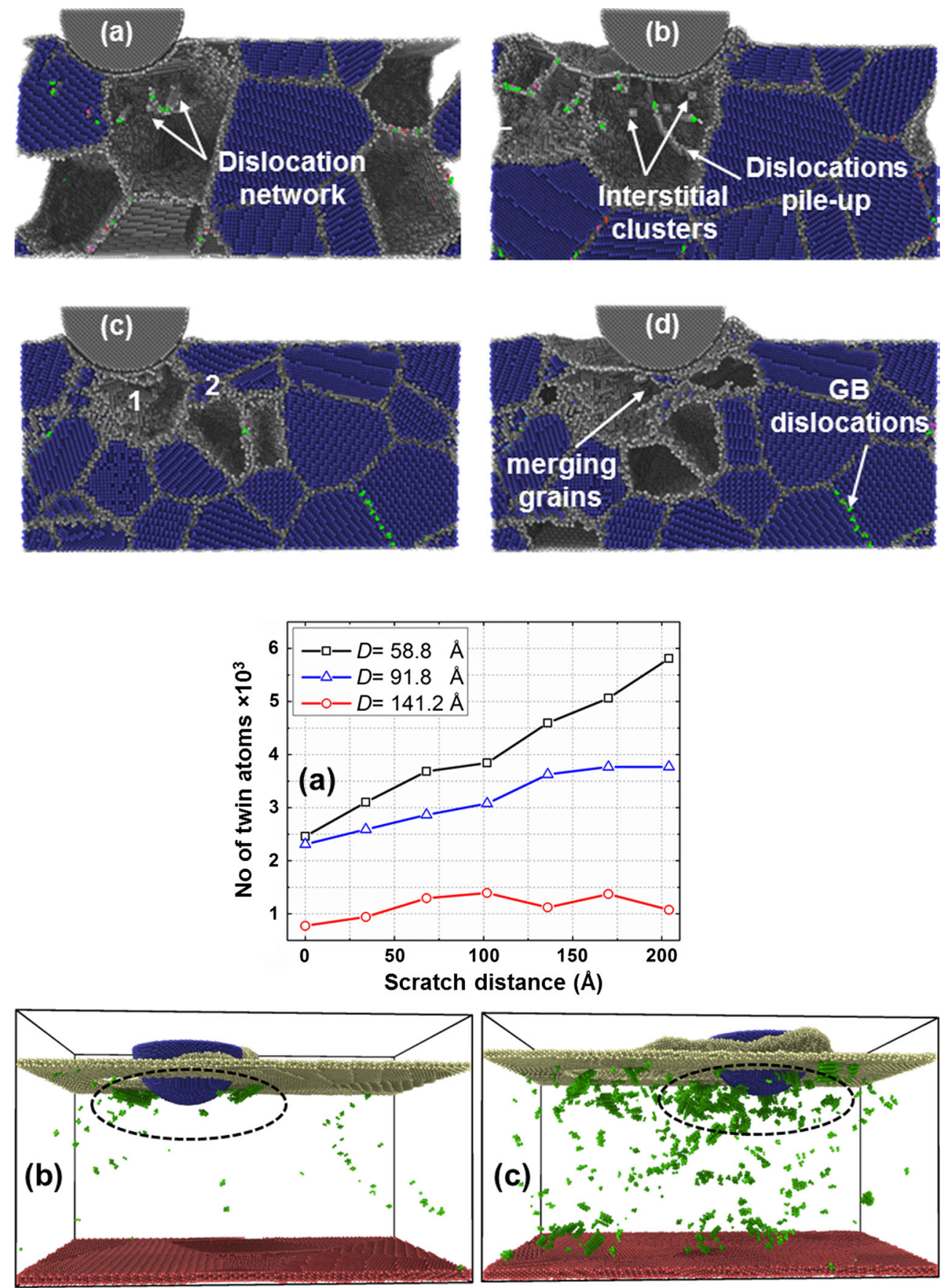

Fig. 8 Variation of number of twin atoms vs. scratch distance for different grain size (a).

Atomistic snapshot showing the twinned atoms (green) for grain sizes b $D=141.2 \AA$, and c $D=58.8 \AA$. Note that lattice and GB atoms are omitted for better visualization. The twinned atoms are identified using CAT [29, 30] (Color figure online) nucleated dislocation does not glide into the grain interior. Instead, dislocation pileup occurs at grain boundaries. Interestingly, we observed a large fraction of twins formed in front of and beneath the tool, while sliding as shown in Fig. $8 \mathrm{~b}$ and the fraction of twins increased with decreasing grain size, Fig. 8. Plasticity via formation of twins has previously been reported for both single crystal [40-43] and polycrystalline iron [44]. It has been shown, for sc-iron, that twinning occurs as a results of disassociation of [111] core of an $(\mathrm{a} / 2)\langle 111\rangle$ dislocation into three $(\mathrm{a} / 6)\langle 111\rangle$ fractional dislocations. While in nanocrystalline metals, the origin of the twinned area is believed to be via the intragranular dislocation glide, although dislocations are absent in the grain interior in normal condition $[45,46]$. The formation of twins in smaller grains can be explained by two mechanisms. Firstly, in sliding, the tip/workpiece interface acts as the source for intragranular dislocations; hence, the formation of twins can be activated. Secondly, Fig. 8b clearly shows that twins are formed in the shape of bundles at GBs and the latter phenomena are plausible since the GBs 
represent regions of high stress as has been illustrated in $[47,48]$. In order to further elucidate this point, we have analyzed the stress using the von Mises relationship

$$
=\sqrt{\frac{\left(\sigma_{x x}-\sigma_{y y}\right)^{2}+\left(\sigma_{y y}-\sigma_{z z}\right)^{2}+\left(\sigma_{z z}-\sigma_{x x}\right)^{2}+6\left(\tau_{x y}^{2}+\tau_{y z}^{2}+\tau_{z x}^{2}\right)}{2}}
$$

where $\sigma_{i j}$ is the atomic stress calculated based on the virial theorem [49]. From Fig. 9, it can be seen that most of the highly stressed regions were located in the vicinity of grain boundaries, while the stress inside the grain is lower than that at the grain boundary. Furthermore, we found that the stress concentrators are higher for small grains in comparison with larger grains, Fig. $9 \mathrm{~b}$.

Besides formation of twins, GBs sliding was also observed as a cooperative process during nanoscratching in the case of smaller grains. Figure 10 illustrates an example of grain boundary sliding observed for the sample with $D=58.8 \AA$ A. Several theoretical [50-53] and experimental [54-56] studies have shown that GBs sliding is one of the primary deformation mechanisms that occur upon grain refinement in nanosized polycrystalline material. In the work of Mishra et al. [18], it was reported that GB sliding was the main deformation mechanism during nanomachining of nanocrystalline silicon carbide.

\subsubsection{Variation of Nanoscratch Parameters with Grain Diameter}

The values of frictional $\left(F_{\mathrm{f}}\right)$ and normal $\left(F_{\mathrm{f}}\right)$ forces were calculated by summing up the total forces exerted on the tool atoms in $\mathrm{x}$ and $\mathrm{z}$ directions, respectively. Figure 11 displays the evolution of $F_{\mathrm{f}}, F_{\mathrm{n}}$ as well as their ratio $\left(\mu=\frac{F_{\mathrm{f}}}{F_{\mathrm{n}}}\right)$ during the scratch process. The simulation results show that the frictional forces start to develop from zero and then saturate after $40 \AA$ scratch distance. Such a development of frictional forces results from the formation of dislocations or material pileup. In contrast, the value of the normal forces suddenly dropped at the start of the scratch stage which can be understood as these forces only are required to keep constant scratch depth and not for further penetrations. We found that the instantaneous value of $F_{\mathrm{f}}$ and $F_{\mathrm{n}}$ varies slightly; hence, there is no clear dependence of them, at least not within the studied range of grain sizes. However, the average values of $F_{\mathrm{f}}, F_{\mathrm{n}}$ and $\mu$ averaged over the last $100 \AA$, listed in Table 1 , are discernibly higher in the case of the single crystal than those of all nc-samples which may be attributed to softening by GBs. The above findings are consistent with previous [15] study of nanomachining of polycrystalline copper and recently by Gao and Urbassek [23].

The scratch hardness, defined as the ratio of frictional force and the horizontal projection of the contact area $A_{\mathrm{p}}^{\mathrm{scr}}$ (Fig. 12), is given by

$H_{\text {scr }}=\frac{F_{\mathrm{f}}}{A_{\mathrm{p}}^{\mathrm{scr}}}$

In Fig. 13, we plot the evolution of scratch hardness during scratch distance corresponding to the five different grain sizes as well as for single-crystal iron. In general, the value of $H_{\text {ind }}$ tends to be more than twice as high as $H_{\text {scr. }}$. As illustrated in Fig. 13(inset), the value of $H_{\text {scr }}$, averaged over last $100 \AA$ of scratch distance, initially increased up to $6.72 \mathrm{GPa}$ at $D=71.1 \AA$ and then gradually decreased with increasing grain size from 71.1 to $141.2 \AA$.

During scratching, material removal caused by the sliding tip is commonly observed. The displaced atoms are accumulated in front of the tool, and some are irregularly distributed sideways forming wedges adjacent to the groove. The structural analysis of the pileup materials reveals that they are composed of small grains and amorphous structure as illustrated in Fig. 14. The wear of workpiece material has been quantified by the specific wear rate, $W_{\mathrm{sp}}$, given by [57]

$W_{\mathrm{sp}}=\frac{V_{\mathrm{T}}}{F_{\mathrm{n}} L}$

where $V_{\mathrm{T}}$ is the wear volume, defined as the number of removed atoms from their perfect position multiplied by bcc atomic volume of iron $11.69 \AA$ using current
Fig. 9 Localized von Mises stress corresponding to grain sizes, a $D=141.2 \AA$ and b $D=58.8 \AA$
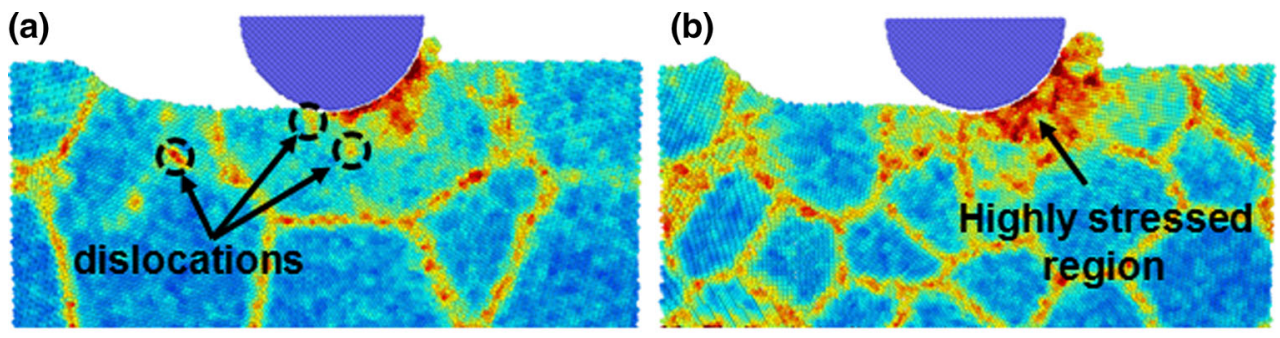

(GPa) 
Fig. 10 MD snapshots illustrating the GBs sliding process of nanoscratched nciron with $D=58.8 \AA$
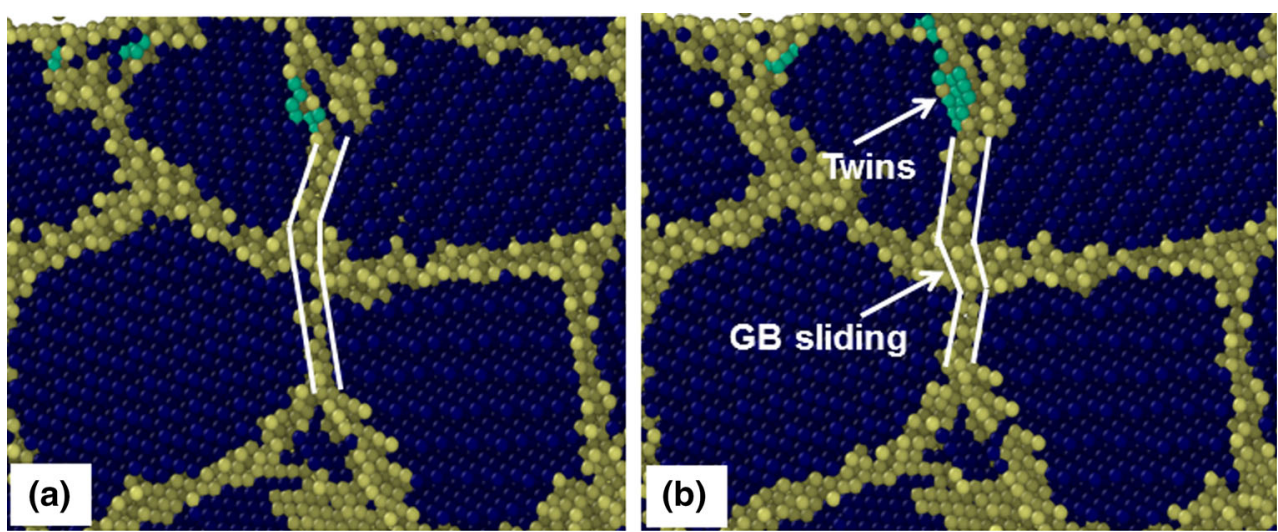

(a)

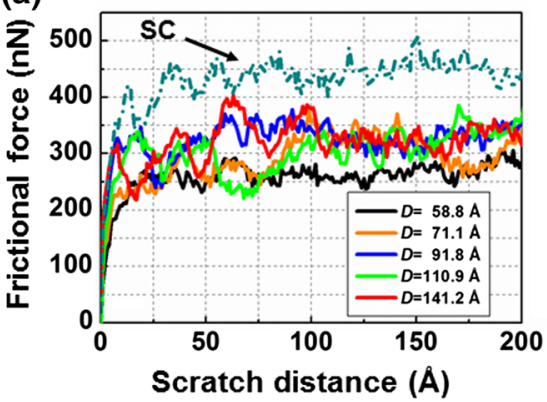

(b) 1

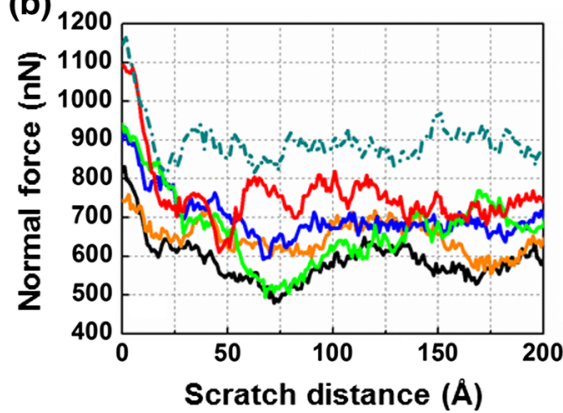

(c)

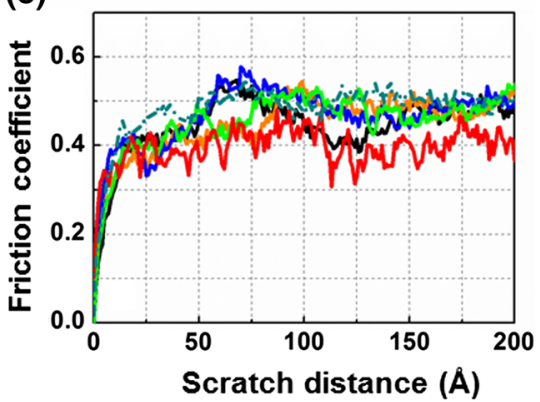

Fig. 11 Grain size dependence of a frictional force, $\mathbf{b}$ normal forces and $\mathbf{c}$ friction coefficient during scratch

Table 1 Summary of nanoscratching characteristics dependence on the scratch depth (d)

\begin{tabular}{llllllc}
\hline$D(\AA)$ & $F_{\mathrm{f}}(\mathrm{nN})$ & $F_{\mathrm{n}}(\mathrm{nN})$ & $\mu$ & $A_{p}^{s c r} \times 10^{3}\left(\AA^{2}\right)$ & $H_{\text {scr }}(\mathrm{GPa})$ & $W_{\text {sp }} \mathrm{nm}^{2} / \mu \mathrm{N}$ \\
\hline 58.8 & 262.31 & 589.54 & 0.44 & 4.67 & 5.59 & 19.63 \\
71.1 & 322.66 & 660.17 & 0.48 & 4.84 & 6.72 & 18.84 \\
91.8 & 324.45 & 682.71 & 0.47 & 5.14 & 6.63 & 15.23 \\
110.9 & 320.43 & 667.91 & 0.48 & 5.07 & 6.37 & 12.88 \\
141.2 & 322.51 & 737.48 & 0.46 & 4.91 & 6.35 & 12.21 \\
$\mathrm{sc}$ & 452.27 & 894.26 & 0.51 & 4.67 & 8.12 & 10.52 \\
\hline
\end{tabular}

The values are obtained by averaging over the last $100 \AA$ scratching distance

Fig. 12 Evolution of contact area during scratching stage
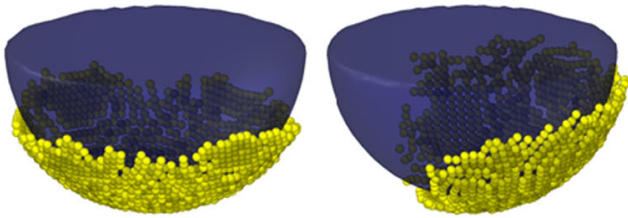

$L=0 \AA$

$L=25 \AA$

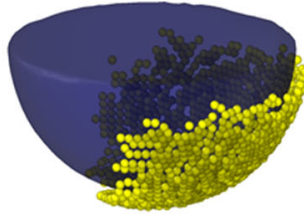

$L=75 \AA$

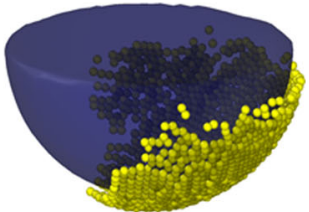

$L=150 \AA$ interatomic potential, $F_{\mathrm{n}}$ is the normal forces, and $L$ is the scratch distance. The results of $W_{\mathrm{sp}}$, calculated by Eq. (8), for various grain diameters as well as for a single crystal, obtained after scratch distance $150 \AA$, are listed in Table 1 . Interestingly, the specific wear rate of nc-samples was higher than that of the sc-sample. This finding is persuasive since the grain boundary leads to softening of the materials as indicated by the higher value of sc-iron compared to ncsamples.

From the above results, it is instructive to comment on the response of nanoindentation and scratching hardness to grain size, since the material properties of nanocrystalline materials vary significantly with grain size. Reducing the grain size of the material below nanometers leads to enhanced material 


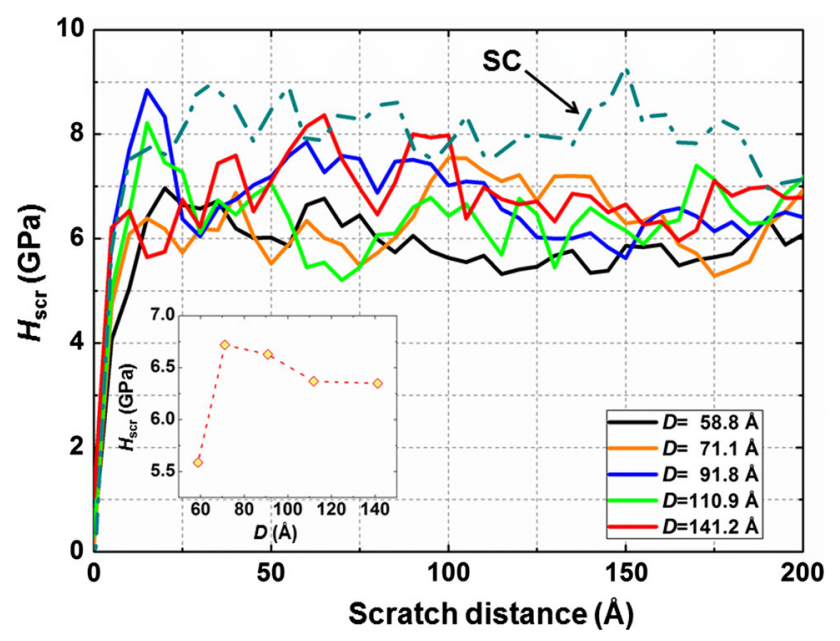

Fig. 13 Grain size dependence of the scratch hardness for different grain sizes $D$. The average value of $H_{\text {scr }}$ versus $D$ (inset)
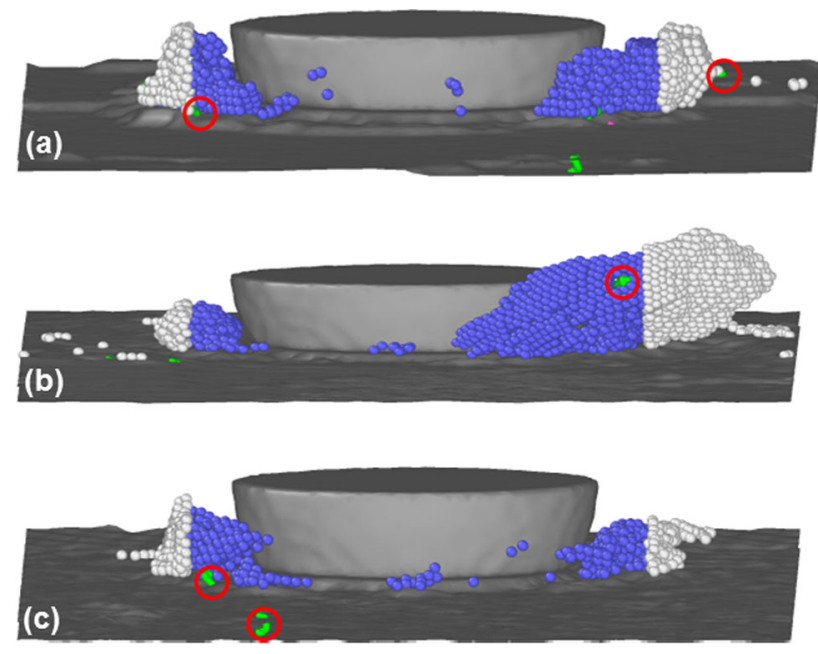

Fig. 14 Frontal view of pileup obtained at scratch distance $100 \AA$ for a $58.8 \AA$, b D $=141.2 \AA$ and c single crystal. Blue atoms represent crystalline and the red circles mark the dislocation climb (Color figure online)

strength according to the Hall-Petch (H-P) relationship and further grain refinement results in lowering of the strength against inverse (H-P) relationship. In close agreement with the previous results of MD simulation of grain size dependence of indentation hardness of polycrystalline copper [36], our indentation hardness results exhibit a continuous decrease with grain refinement, i.e., inverse H-P. Whereas the scratch hardness does not show clear dependence on the grain size since its value slightly varies with $D$. In order to examine if there is a direct correlation between these two parameters with intrinsic material properties, we carried out MD simulation of uniaxial tensile tests at a strain rate $5 \times 10^{8} \mathrm{~s}^{-1}$ of nanocrystalline iron. Figure 15a shows the typical stressstrain curves of polycrystalline iron with different grain sizes. The variation of flow stress, averaged over $25-30 \%$ strain, with grain size clearly indicates the increase in the strength to its maximum at grain diameter $D=110.9 \AA$ and then decrease as illustrated in Fig. 15b. Hence, both inverse H-P and $\mathrm{H}-\mathrm{P}$ relationship are observed with this range of grain sizes in good agreement with previous MD simulations of nciron [51]. Therefore, we can conclude that these two parameters are tribo-parameters dependent, rather than intrinsic material property dependent.

\subsubsection{On the Correlation Between Tip Diameter, Grain Size and Wear Characteristic}

Material behavior, during nanoindentation and nanoscratch testing, is strongly dependent on the geometrical shape and surface topography of the tip. Thus, we investigated the effect of the tip shape on the wear/friction of nc-iron by changing tip radii while keeping the grain size of $D=91.8 \AA$ and scratch depth of $d=10 \AA$ fixed. The simulation results of nanoscratch of the sample using different tip sizes are presented in Fig. 16. It can be seen that the average values of both frictional and normal forces increase with tip size. However, the increase in the normal force is more pronounced. Furthermore, we noticed that the friction coefficient initially decreases and then saturates above tip size $R=45 \AA$, as shown in Fig. 16 .

From the above results, it is interesting to see that some nanoscratching parameters, in particular $F_{\mathrm{f}}$ and $\mu$, converge when the grain size is quantitatively comparable with the tip size. Indeed, this critical value of tip diameter $(90 \AA)$ is close to the average grain size $D=91.8 \AA$. Consequently, these findings suggest that there is a correlation between the tip and grain size with the scratch parameters.

Contrary to the recent MD results of scratch hardness of nc-iron [23] in which the scratch hardness slightly increases with increasing tip size, our MD results show that the value of scratch hardness slightly varies with tip size. This inconsistency may be attributed to different simulation parameters such as tip size, grain size and scratch depth. Finally, from the results of friction coefficient and specific wear rate, one can see that improved friction properties can be achieved by tuning the size of the tip to be relatively comparable with the grain size.

With respect to the variation of the wear with tip size, we noticed that the change of the tip radius has strong influence on the $W_{\mathrm{sp}}$ calculated using Eq. (8). As illustrated in Table 2, increasing the tip radius from 25 to $60 \AA$ leads to a significant decrease in the value of $W_{\mathrm{sp}}$ by about $81.5 \%$.

\subsubsection{Variation of Nanoscratching Parameters with Scratch Depth}

We examined the dependence of scratch characteristic on the scratch depth for a specific nc-sample with 
Fig. 15 Stress versus strain curves for various grain sizes obtained at a strain rate of $5 \times 10^{8} \mathrm{~s}^{-1}(\mathbf{a})$, and the variation of flow stress with grain size (b). The yellow line marks the transition between conventional to inverse $\mathrm{H}-\mathrm{P}$ relationship (Color figure online)

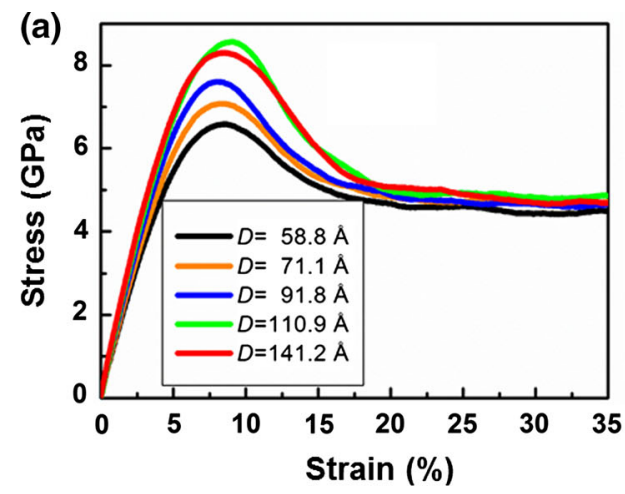

(b)

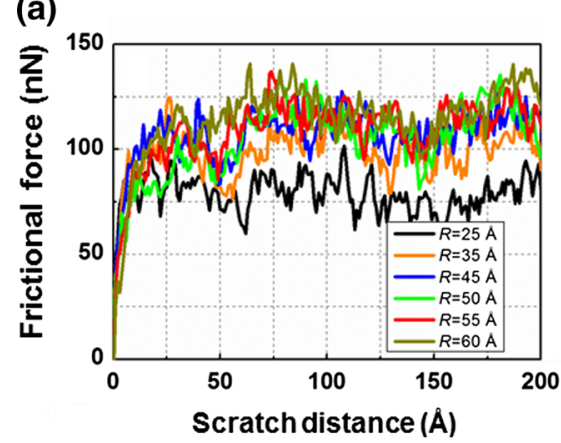

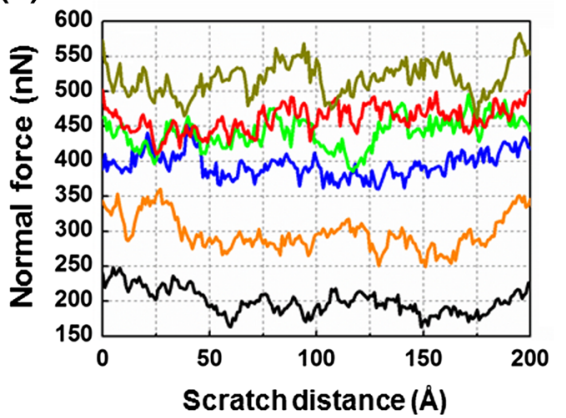

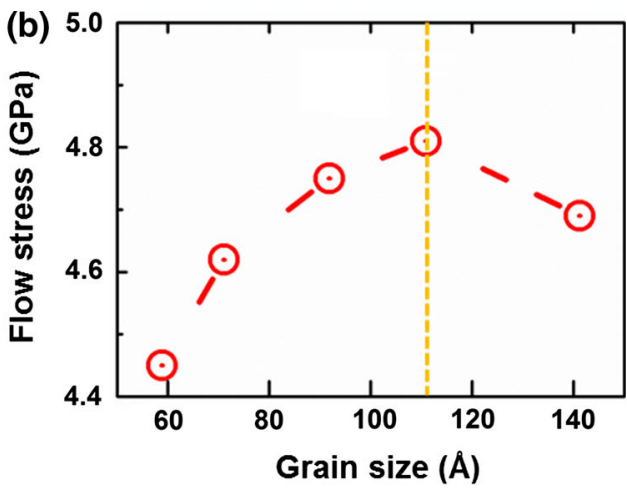

(c)

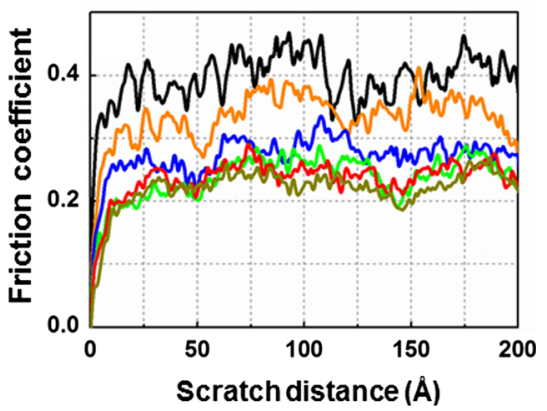

Fig. 16 Tip radius $(R)$ dependence of nanoscratch properties a frictional force, $\mathbf{b}$ normal forces and $\mathbf{c}$ friction coefficient during scratch

Table 2 Summary of tip radius dependence of nanoscratching characteristics

\begin{tabular}{lclllll}
\hline$R(\AA)$ & $F_{\mathrm{f}}(\mathrm{nN})$ & $F_{\mathrm{n}}(\mathrm{nN})$ & $\mu$ & $A_{p}^{s c r}\left(\AA^{2}\right)$ & $H_{\mathrm{scr}}(\mathrm{GPa})$ & $W_{\mathrm{sp}}\left(\mathrm{nm}^{2} \mu \mathrm{N}^{-1}\right)$ \\
\hline 25 & 75.79 & 192.50 & 0.39 & 1252 & 6.05 & 7.27 \\
35 & 99.81 & 285.87 & 0.34 & 1548 & 6.44 & 4.27 \\
45 & 109.92 & 385.61 & 0.28 & 1761 & 6.24 & 2.33 \\
50 & 108.57 & 439.38 & 0.23 & 1742 & 6.23 & 1.88 \\
55 & 113.21 & 468.23 & 0.24 & 1722 & 6.57 & 1.66 \\
60 & 113.91 & 517.11 & 0.24 & 1700 & 6.70 & 1.34 \\
\hline
\end{tabular}

The values are obtained by averaging over the last $100 \AA$ scratching distance
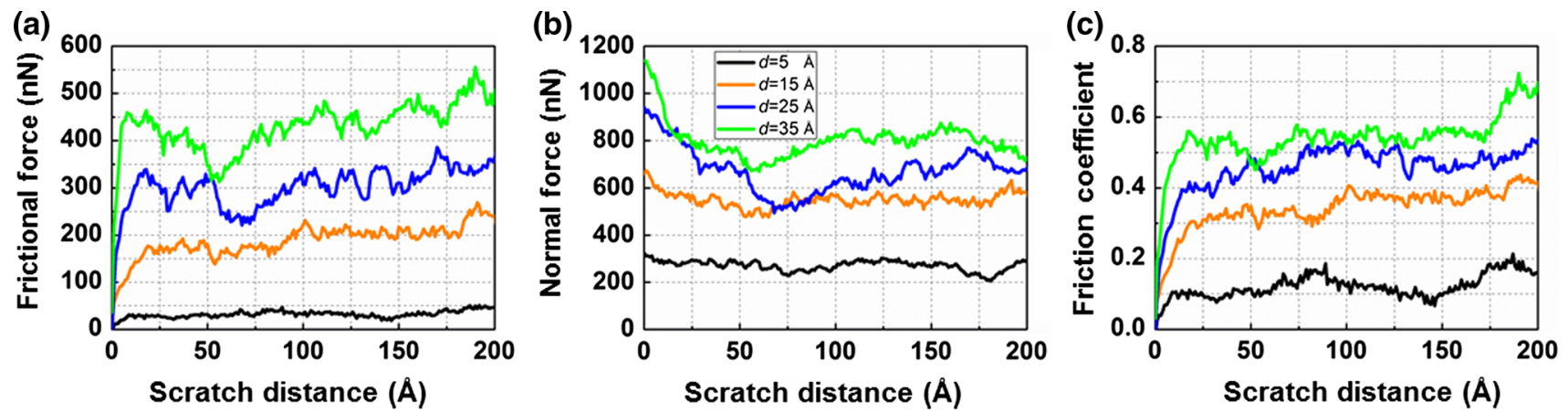

Fig. 17 Scratch depth $(d)$ dependence of nanoscratch properties a frictional force, $\mathbf{b}$ normal forces and $\mathbf{c}$ friction coefficient

$D=110.9 \AA$. We varied the scratch depth between 5 and $35 \AA$, while the tip size was kept fixed $(R=100 \AA)$. The MD results show that the scratch characteristics vary greatly with the scratch depth. Figure 17 displays the evolution of frictional forces, normal forces and friction coefficient for different scratch depths. The values of $F_{\mathrm{f}}, F_{\mathrm{n}}$ and $\mu$ averaged 
Table 3 Summary of nanoscratching characteristics dependence on the scratch depth (d) at $R=100 \AA$

\begin{tabular}{lclllll}
\hline$d(\AA)$ & $F_{\mathrm{f}}(\mathrm{nN})$ & $F_{\mathrm{n}}(\mathrm{nN})$ & $\mu$ & $A_{p}^{s c r}\left(\AA^{2}\right)$ & $H_{\text {scr }}(\mathrm{GPa})$ & $W_{\mathrm{sp}}\left(\mathrm{nm}^{2} \mu \mathrm{N}^{-1}\right)$ \\
\hline 5 & 31.41 & 275.58 & 0.11 & 618.3 & 5.18 & 0.28 \\
15 & 205.60 & 547.01 & 0.38 & 3273.6 & 6.28 & 6.16 \\
25 & 320.43 & 667.91 & 0.48 & 5077.2 & 6.37 & 12.88 \\
35 & 450.37 & 823.19 & 0.55 & 6868.7 & 6.54 & 27.59 \\
\hline
\end{tabular}

The values are obtained by averaging over the last $100 \AA$
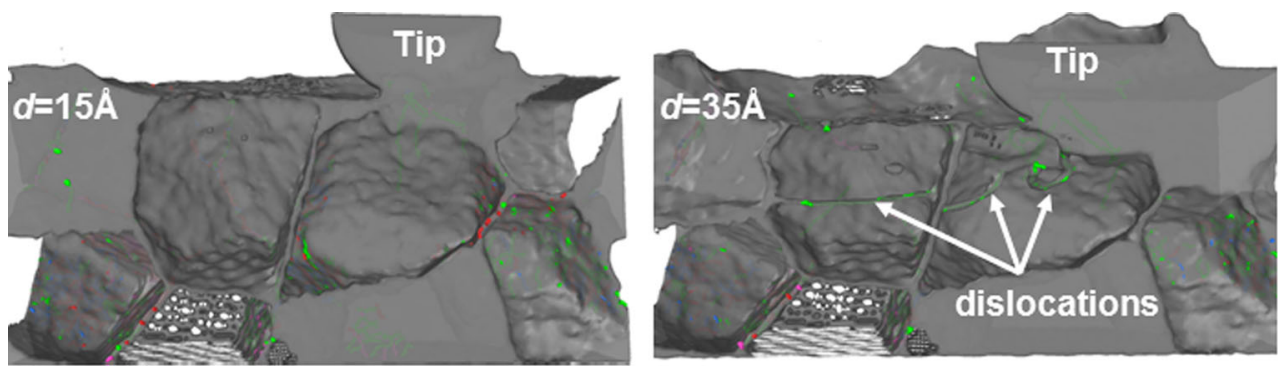

Fig. 18 MD snapshot showing the grains directly beneath the tip at different scratch depths. Atoms are omitted to expose grain interior. Note that the absence of dislocation in interior of grain at $d=15 \AA$ over the 100 A scratching length are summarized in Table 3. It is clear that frictional and normal forces are higher for larger scratch depths. As the depth increases, the tip displacement through the substrate is more difficult due to the resistance to tip motion from the accumulated material in front of it. Similarly, the calculated friction coefficient increased by about $80 \%$ when the scratch depth increased from 5 to $35 \AA$. Similar trends of $F_{\mathrm{f}}, F_{\mathrm{n}}$ and $\mu$ with scratch depth have been observed for MD studies of iron single crystal $[19,21]$. The increase in the friction coefficient can be attributed to the increase in the dislocation density with increasing scratch depth as illustrated in Fig. 18. Our MD results are in good agreement with the recent experimental work of scratching of pure iron [58]. The large increase in the friction coefficient upon increasing the scratch depth is clear evidence of transition of wear mechanism from plowing to cutting. The transition in the wear mechanisms with increasing scratch depth has been experimentally observed in scratching of $\mathrm{Ni}$ and $\mathrm{Cu}$ by AFM [59].

\section{Conclusion}

We have performed large-scale molecular dynamics simulations of single-asperity nanoscratch of nanocrystalline iron to investigate the effect of grain size, tip radius and scratch depth on its wear behavior. Five samples with different grain sizes were considered as well as a singlecrystal sample for the purpose of comparison. In all studied samples, the deformation mechanism was mediated by dislocation nucleation within grain interior as well as grain boundary dislocations. However, the subsequent plasticity mechanism is different depending on the grain size. While the dislocation propagation is associated with formation of nanovoids and interstitial clusters in the case of larger grain size, the formation of twins at the grain boundary was dominating for smaller clusters. This behavior was attributed to the transition of dislocation movement from smooth (larger grain) to rough (smaller grain) during scratching.

Our atomistic results indicated that the values of both the frictional and normal forces slightly increase with increasing grain size. However, the values were still lower than those of iron single crystal.

The indentation/scratch hardness of the nc-iron has been calculated during indentation and scratching stages, and in general, the magnitude of indentation hardness decreases with decreasing grain size, while the scratch hardness dependence on the grain size was considerably small. Moreover, the wear loss of each sample was quantified in terms of the specific wear rate, and the simulation results showed that the wear rate increased with increasing grain size. However, they were always higher as compared to the single-crystal material. Additionally, the variation of scratch parameters with contact size and scratch depth dependence of scratch parameters were examined, and the results show that the wear/friction behavior of nc-samples could be controlled by these factors.

Acknowledgements We are grateful to the Swedish National Infrastructure for Computing (SNIC) at the National Supercomputer Centre in Linköping for providing computational resources under the Project SNIC2016-1-354.

Open Access This article is distributed under the terms of the Creative Commons Attribution 4.0 International License (http://crea tivecommons.org/licenses/by/4.0/), which permits unrestricted use, distribution, and reproduction in any medium, provided you give appropriate credit to the original author(s) and the source, provide a link to the Creative Commons license, and indicate if changes were made. 


\section{References}

1. Beake, B.D., Harris, A.J., Liskiewicz, T.W.: Review of recent progress in nanoscratch testing. Tribol. Mater. Surf. Interfaces 7, 87-96 (2013)

2. Tiwari, A.: Applied Nanoindentation in Advanced Materials. Wiley, New York (2016)

3. Ikawa, N., Shimada, S., Tanaka, H.: Minimum thickness of cut in micromachining. Nanotechnology 3, 6 (1992)

4. Fang, T.-H., Weng, C.-I.: Three-dimensional molecular dynamics analysis of processing using a pin tool on the atomic scale. Nanotechnology 11, 148 (2000)

5. Mishra, M., Szlufarska, I.: Dislocation controlled wear in single crystal silicon carbide. J. Mater. Sci. 48, 1593-1603 (2012)

6. Zhang, L., Huang, H., Zhao, H., Ma, Z., Yang, Y., Hu, X.: The evolution of machining-induced surface of single-crystal FCC copper via nanoindentation. Nanoscale Res. Lett. 8, 1-13 (2013)

7. Zhang, L., Zhao, H., Dai, L., Yang, Y., Du, X., Tang, P., Zhang, L.: Molecular dynamics simulation of deformation accumulation in repeated nanometric cutting on single-crystal copper. RSC Adv. 5, 12678-12685 (2015)

8. Wang, Q., Bai, Q., Chen, J., Sun, Y., Guo, Y., Liang, Y.: Subsurface defects structural evolution in nano-cutting of single crystal copper. Appl. Surf. Sci. 344, 38-46 (2015)

9. Wang, Q., Bai, Q., Chen, J., Su, H., Wang, Z., Xie, W.: Influence of cutting parameters on the depth of subsurface deformed layer in nano-cutting process of single crystal copper. Nanoscale Res. Lett. 10, 1-8 (2015)

10. Hall, E.O.: The deformation and ageing of mild steel: III discussion of results. Proc. Phys. Soc. Sect. B 64, 747 (1951)

11. Petch, N.J.: The cleavage strength of polycrystals. J. Iron Steel Inst. 174, 25-28 (1953)

12. Chokshi, A.H., Rosen, A., Karch, J., Gleiter, H.: On the validity of the Hall-Petch relationship in nanocrystalline materials. Scr. Metall. 23, 1679-1683 (1989)

13. Conrad, H., Narayan, J.: Mechanism for grain size softening in nanocrystalline Zn. Appl. Phys. Lett. 81, 2241-2243 (2002)

14. Junge, T., Molinari, J.-F.: Plastic activity in nanoscratch molecular dynamics simulations of pure aluminium. Int. J. Plast 53, 90-106 (2014)

15. Shi, J., Wang, Y., Yang, X.: Nano-scale machining of polycrystalline coppers-effects of grain size and machining parameters. Nanoscale Res. Lett. 8, 500 (2013)

16. Goel, S., Luo, X., Agrawal, A., Reuben, R.L.: Diamond machining of silicon: a review of advances in molecular dynamics simulation. Int. J. Mach. Tools Manuf. 88, 131-164 (2015)

17. Mo, Y., Stone, D., Szlufarska, I.: Strength of ultrananocrystalline diamond controlled by friction of buried interfaces. J. Phys. Appl. Phys. 44, 405401 (2011)

18. Mishra, M., Tangpatjaroen, C., Szlufarska, I.: Plasticity-controlled friction and wear in nanocrystalline SiC. J. Am. Ceram. Soc. 97, 1194-1201 (2014)

19. Mulliah, D., Kenny, S.D., McGee, E., Smith, R., Richter, A., Wolf, B.: Atomistic modelling of ploughing friction in silver, iron and silicon. Nanotechnology 17, 1807-1818 (2006)

20. Lu, C., Gao, Y., Deng, G.Y., Michal, G., Huynh, N.N., Liu, X.H., Tieu, A.K.: Atomic-scale anisotropy of nanoscratch behavior of single crystal iron. Wear 267, 1961-1966 (2009)

21. Gao, Y., Ruestes, C.J., Urbassek, H.M.: Nanoindentation and nanoscratching of iron: atomistic simulation of dislocation generation and reactions. Comput. Mater. Sci. 90, 232-240 (2014)

22. Gao, Y., Brodyanski, A., Kopnarski, M., Urbassek, H.M.: Nanoscratching of iron: a molecular dynamics study of the influence of surface orientation and scratching direction. Comput. Mater. Sci. 103, 77-89 (2015)

23. Gao, Y., Urbassek, H.M.: Scratching of nanocrystalline metals: a molecular dynamics study of Fe. Appl. Surf. Sci. 389, 688-695 (2016)

24. Voronoi, G.: Nouvelles applications des paramètres continus à la théorie des formes quadratiques. Deuxième mémoire. Recherches sur les parallélloèdres primitifs. J. Für Reine Angew. Math. Crelles J. 1908, 198-287 (2009)

25. Hirel, P.: Atomsk: a tool for manipulating and converting atomic data files. Comput. Phys. Commun. 197, 212-219 (2015)

26. Mendelev, M.I., Han, S., Srolovitz, D.J., Ackland, G.J., Sun, D.Y., Asta, M.: Development of new interatomic potentials appropriate for crystalline and liquid iron. Philos. Mag. 83, 3977-3994 (2003)

27. Banerjee, S., Naha, S., Puri, I.K.: Molecular simulation of the carbon nanotube growth mode during catalytic synthesis. Appl. Phys. Lett. 92, 233121 (2008)

28. Stukowski, A., Albe, K.: Extracting dislocations and non-dislocation crystal defects from atomistic simulation data. Model. Simul. Mater. Sci. Eng. 18, 085001 (2010)

29. Stukowski, A., Arsenlis, A.: On the elastic-plastic decomposition of crystal deformation at the atomic scale. Model. Simul. Mater. Sci. Eng. 20, 035012 (2012)

30. Stukowski, A.: Structure identification methods for atomistic simulations of crystalline materials. Model. Simul. Mater. Sci. Eng. 20, 045021 (2012)

31. Möller, J.J., Bitzek, E.: BDA: a novel method for identifying defects in body-centered cubic crystals. MethodsX 3, 279-288 (2016)

32. Tschopp, M.A., Tucker, G.J., McDowell, D.L.: Atomistic simulations of tension-compression asymmetry in dislocation nucleation for copper grain boundaries. Comput. Mater. Sci. 44, 351-362 (2008)

33. Tschopp, M.A., McDowell, D.L.: Dislocation nucleation in $\Sigma 3$ asymmetric tilt grain boundaries. Int. J. Plast. 24, 191-217 (2008)

34. Guleryuz, E., Mesarovic, S.: Dislocation nucleation on grain boundaries: low angle twist and asymmetric tilt boundaries. Crystals 6, 77 (2016)

35. Ziegenhain, G., Urbassek, H.M., Hartmaier, A.: Influence of crystal anisotropy on elastic deformation and onset of plasticity in nanoindentation: a simulational study. J. Appl. Phys. 107, 061807 (2010)

36. Liu, X., Yuan, F., Wei, Y.: Grain size effect on the hardness of nanocrystal measured by the nanosize indenter. Appl. Surf. Sci. 279, 159-166 (2013)

37. Ma, X.-L., Yang, W.: Molecular dynamics simulation on burst and arrest of stacking faults in nanocrystalline $\mathrm{Cu}$ under nanoindentation. Nanotechnology 14, 1208 (2003)

38. Feichtinger, D., Derlet, P.M., Van Swygenhoven, H.: Atomistic simulations of spherical indentations in nanocrystalline gold. Phys. Rev. B 67, 024113 (2003)

39. Marian, J., Cai, W., Bulatov, V.V.: Dynamic transitions from smooth to rough to twinning in dislocation motion. Nat. Mater. 3, 158-163 (2004)

40. Ersland, C.H., Vatne, I.R., Thaulow, C.: Atomistic modeling of penny-shaped and through-thickness cracks in bcc iron. Model. Simul. Mater. Sci. Eng. 20, 075004 (2012)

41. Al-Motasem, A.T., Mai, N.T., Choi, S.T., Posselt, M.: Atomistic study on mixed-mode fracture mechanisms of ferrite iron interacting with coherent copper and nickel nanoclusters. J. Nucl. Mater. 472, 20-27 (2016)

42. Ojha, A., Sehitoglu, H., Patriarca, L., Maier, H.J.: Twin nucleation in Fe-based bcc alloys-modeling and experiments. Model. Simul. Mater. Sci. Eng. 22, 075010 (2014) 
43. Machová, A., Beltz, G.E., Chang, M.: Atomistic simulation of stacking fault formation in bcc iron. Model. Simul. Mater. Sci. Eng. 7, 949 (1999)

44. Gunkelmann, N., Tramontina, D.R., Bringa, E.M., Urbassek, H.M.: Morphological changes in polycrystalline Fe after compression and release. J. Appl. Phys. 117, 085901 (2015)

45. Zhu, Y.T., Huang, J.Y., Gubicza, J., Ungár, T., Wang, Y.M., Ma, E., Valiev, R.Z.: Nanostructures in Ti processed by severe plastic deformation. J. Mater. Res. 18, 1908-1917 (2003)

46. Shan, Z., Stach, E.A., Wiezorek, J.M.K., Knapp, J.A., Follstaedt, D.M., Mao, S.X.: Grain Boundary-mediated plasticity in nanocrystalline nickel. Science 305, 654-657 (2004)

47. Niewczas, M.: Chapter 75 dislocations and twinning in face centred cubic crystals. In: Dislocations in Solids, vol. 13, pp. 263-364. Elsevier, Amsterdam (2007)

48. Tari, V., Rollett, A.D., Kadiri, H.E., Beladi, H., Oppedal, A.L., King, R.L.: The effect of deformation twinning on stress localization in a three dimensional TWIP steel microstructure. Model. Simul. Mater. Sci. Eng. 23, 045010 (2015)

49. Thompson, A.P., Plimpton, S.J., Mattson, W.: General formulation of pressure and stress tensor for arbitrary many-body interaction potentials under periodic boundary conditions. J. Chem. Phys. 131, 154107 (2009)

50. Schiøtz, J., Jacobsen, K.W.: A maximum in the strength of nanocrystalline copper. Science 301, 1357-1359 (2003)

51. Jeon, J.B., Lee, B.-J., Chang, Y.W.: Molecular dynamics simulation study of the effect of grain size on the deformation behavior of nanocrystalline body-centered cubic iron. Scr. Mater. 64, 494-497 (2011)

52. Van Swygenhoven, H., Derlet, P.M.: Grain-boundary sliding in nanocrystalline fcc metals. Phys. Rev. B. 64, 224105 (2001)

53. Yamakov, V., Wolf, D., Phillpot, S.R., Mukherjee, A.K., Gleiter, H.: Deformation-mechanism map for nanocrystalline metals by molecular-dynamics simulation. Nat. Mater. 3, 43-47 (2004)

54. Sergueeva, A.V., Mara, N.A., Krasilnikov, N.A., Valiev, R.Z., Mukherjee, A.K.: Cooperative grain boundary sliding in nanocrystalline materials. Philos. Mag. 86, 5797-5804 (2006)

55. Ivanisenko, Y., Kurmanaeva, L., Weissmueller, J., Yang, K., Markmann, J., Rösner, H., Scherer, T., Fecht, H.-J.: Deformation mechanisms in nanocrystalline palladium at large strains. Acta Mater. 57, 3391-3401 (2009)

56. Kumar, K.S., Suresh, S., Chisholm, M.F., Horton, J.A., Wang, P.: Deformation of electrodeposited nanocrystalline nickel. Acta Mater. 51, 387-405 (2003)

57. Morris, S., Wood, R.J.K., Harvey, T.J., Powrie, H.E.G.: Electrostatic charge monitoring of unlubricated sliding wear of a bearing steel. Wear 255, 430-443 (2003)

58. Pöhl, F., Hardes, C., Theisen, W.: Chair of materials technology, Ruhr-Universität Bochum, Bochum, Germany: scratch behavior of soft metallic materials. AIMS Mater. Sci. 3, 390-403 (2016)

59. Islam, S., Ibrahim, R.N.: Mechanism of abrasive wear in nanomachining. Tribol. Lett. 42, 275 (2011) 